\title{
Broadband and Highly Efficient sub-THz Reflective Polarization Converter based on Z-shaped Metasurface
}

\author{
Meng $\mathrm{Li}^{\mathrm{a}}$, Feng Lan, Ziqiang Yang, Yaxin Zhang, Zongjun Shi, Minglei Shi, \\ Hongen Su and Feng Luo
}

Terahertz Research Centre, School of Physical Electronics, University of Electronic Science and
Technology of China

No.4, Section 2, North Jianshe Road, Chengdu 610054, P. R China

ae-mail: lemon_meng123@163.com

Keywords: Broadband, Reflective, Polarization, Converter, Metasurface

\begin{abstract}
In this paper, a broadband and highly efficient reflective polarization converter based on Z-shaped metasurface is demonstrated at sub-terahertz ( $\mathrm{THz}$ ) frequencies. The polarizer can convert a linearly polarized (LP) wave to its cross-polarized wave at three resonant frequencies, which can also convert a LP wave to a circularly polarized(CP) wave at other two frequencies. The polarization conversion efficiency for normal incidence larger than $80 \%$ is obtained from $0.116 \mathrm{THz}$ to $0.26 \mathrm{THz}$. The surface current distributions are demonstrated to investigate the physical mechanism. Furthermore, the performance under oblique incidence shows the broad bandwidth can be sustained as incident angle increases to $45^{\circ}$. The polarizer could be useful for enhancing the efficiency of polarization conversion in $\mathrm{THz}$ regime.
\end{abstract}

\section{Introduction}

Polarization converter is an important device of EM waves [1], with the deepening of the research, the polarizer at $\mathrm{THz}$ frequencies has drawn tremendous attention due to its large range of applications in the area of imaging and communications [2] [3]. Conventional polarization converters use birefringence to realize phase retardation, and they often have large thickness and complicated structure[4] [5].

Recently, metamaterials(MMs) have captured extremely attention due to their unique optical properties[6], such as negative refractive index, optical activity and circular dichroism. Metasurfaces[7] are two-dimensional planar MMs, taking the advantage of their ultra-thin features, devices with miniaturization, high integration and good performance can be designed. Up to date, linear or circular polarization converters based on MMs at microwave frequencies have been demonstrated, such as E-shaped[8], L-shaped[9] and F-shaped [10]polarizes, which have multiband properties. However, the polarization conversion only take place in several resonant frequencies with these devices. In another approach, a polarizer with broad bandwidth based on cut-wire was demonstrated[11]. Unfortunately, the development at $\mathrm{THz}$ frequencies is restricted due to the lack of suitable materials and huge fabrication challenges. Previously, Grady et al. have designed an ultrathin broadband linear polarization converter at $\mathrm{THz}$ frequencies[12], after that the split-ring resonators (SRRs) and disk resonators (together henceforth referred to as DSRRs) structure were proposed which can realize broadband polarization conversion in $\mathrm{THz}$ regime [13]. Another reflective linear polarization converter in the near-infrared region was proposed based on ellipse-shaped MMs[14]. These methods make it possible to design a polarization converter at $\mathrm{THz}$ frequencies. However, designing a polarizer with higher efficiency and broader operating bandwidth remains an issue.

In this paper, a broadband and highly efficient polarizer based on Z-shaped metasurface is proposed at sub-THz frequencies. The structure consists of three layers, which can realize a LP wave converts to its cross-polarization wave at the frequencies of $0.123,0.18$ and $0.248 \mathrm{THz}$, simultaneously, the LP wave converts to a CP wave at the frequencies of 0.107 and $0.273 \mathrm{THz}$. The 
polarization conversion efficiency for normal incidence larger than $80 \%$ can be obtained from $0.116 \mathrm{THz}$ to $0.26 \mathrm{THz}$. The proposed structure has simple geometry, broad working bandwidth and high efficiency compared with previous designs.

\section{Design and Simulation}

The stereogram of the polarizer and the diagram of unit cell are demonstrated on Fig.1, respectively. The structure is consisted of three layers, the top layer is the Z-shaped metasurface, the two side arms are perpendicular to the central cut-wire, respectively. The middle layer is dielectric substrate and the bottom layer is full metallic sheet. The metal material is aluminum (with a conductivity of $3.8 \times \mathrm{S} / \mathrm{m}$ ), and the dielectric substrate is quartz (with a dielectric constant 4.41 and a loss tangent 0.0004 ), the thickness for this layer is t. The angle of deflection $\alpha$ is $30^{\circ}$, which can ensure the coupling to be strongest. Optimized geometric parameters are as follows: $\mathrm{p}=550 \mu \mathrm{m}$, $\mathrm{w}=410 \mu \mathrm{m}, \mathrm{t}=180 \mu \mathrm{m}, \mathrm{m}=190 \mu \mathrm{m}, \mathrm{d}=30 \mu \mathrm{m}, \mathrm{g}=90 \mu \mathrm{m}$.
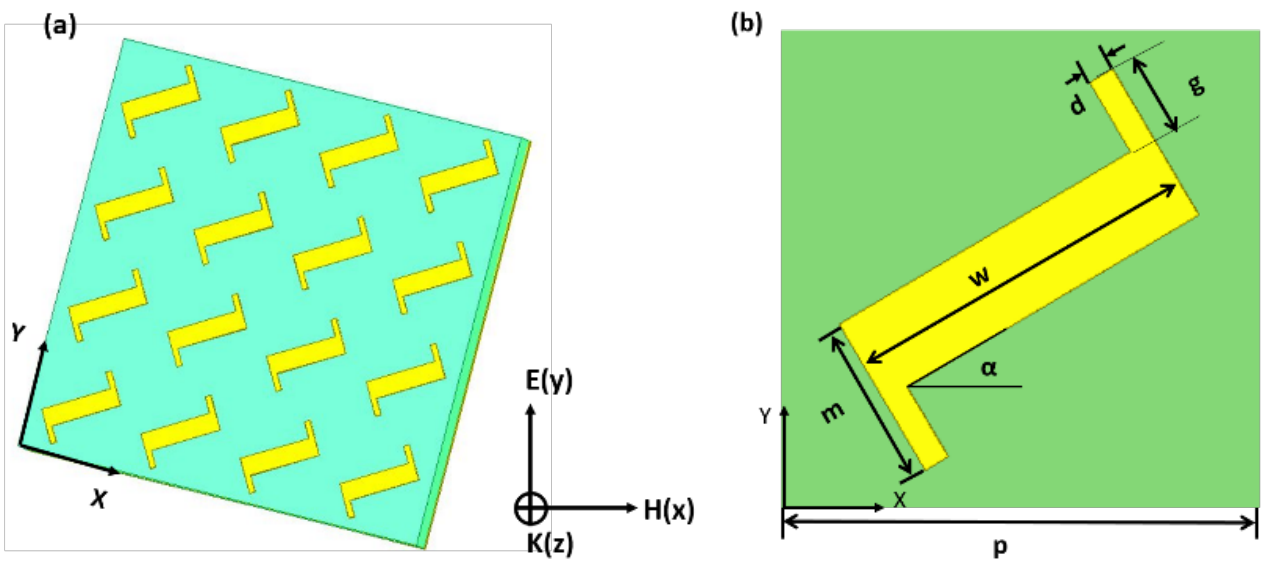

Fig. 1. (a) stereogram of the proposed polarizer (b) the diagram of unit cell

To better understand the polarization conversion behind the designed structure, we define $\mathrm{r}_{y y}=E_{y r} / E_{y i}$ and $\mathrm{r}_{x y}=E_{x r} / E_{y i}$ to represent the reflection ratio y-to-y and y-to- $\mathrm{x}$ polarization conversions, respectively. The subscripts $i$ and $r$ represent incident and reflected EM waves. The subscripts $\mathrm{x}$ and $\mathrm{y}$ indicate the polarization patterns of EM waves. Define the polarization conversion ratio $(P C R)$ as $P C R=\left|r_{x y}\right|^{2} /\left(\left|r_{x y}\right|^{2}+\left|r_{y y}\right|^{2}\right)$ for y-polarization incidence waves. Phase differences between the $\mathrm{y}$ and $\mathrm{x}$ components of the reflected EM wave are defined as $\Delta \phi_{x y}, \Delta \phi_{x y}$ can take arbitrary within $\left[-180^{\circ},+180^{\circ}\right]$ depending on the frequency, meaning that all polarization states (circular, linear, elliptic) are possible for the reflected beam. $\Delta \phi_{x y}=0^{\circ}$ (or $180^{\circ}$ ) indicates a linearly polarized state. The LP wave converts to a CP wave at the situation of $\left|r_{x y}\right|=\left|r_{y y}\right|$ and $\Delta \phi_{x y}=90^{\circ}$, when $\Delta \phi_{x y}=+90^{\circ}$, which indicates a left-handed circularly polarized (LCP) wave, and $\Delta \phi_{x y}=-90^{\circ}$, which indicates a right-handed circularly polarized (RCP)wave. Others are elliptically polarized waves.

\section{Results and discussion}

Finite integral method was used to analysis the polarization conversion of the proposed device. Fig. 2(a) shows the simulated results of reflectance when the incident $\mathrm{THz}$ beam is linearly polarized. From the Fig. 2(a), one can see that the structure can realize $y$-to-x polarization conversion over a broad bandwidth, this is mainly because the two side arms have strengthened the internal coupling of each unit which can results in multiple resonances. The bandwidth of the reflectance $\left|r_{x y}\right|^{2}$ is over $80 \%$ from 0.116 to $0.26 \mathrm{THz}$, and $\left|r_{x y}\right|^{2}$ is $0.898,0.945$ and 0.899 at the 
frequencies of $0.123,0.18$ and $0.248 \mathrm{THz}$. Meanwhile, the reflectance $\left|\mathrm{r}_{y y}\right|^{2}$ are almost zero at the three resonant frequencies, suggesting there are almost no co-polarization conversion. The PCR are very close to 1 at the three resonant frequencies, as shown in Fig. 2 (b), which means nearly all energy of y-polarized incident wave is converted to x-polarized ones. Fig. 2 (c) shows the ratio of $\left|\mathrm{r}_{x y}\right| /\left|r_{y y}\right|$ and the relative phase difference $\Delta \phi_{x y}$. The co-polarization and cross-polarization present the same value at the frequencies of 0.107 and $0.273 \mathrm{THz}$, meanwhile $\Delta \phi_{x y}$ is very nearly $+90^{\circ}$ at the frequency of $0.107 \mathrm{THz}$, which indicates an LCP wave, and $\Delta \phi_{x y}$ is very nearly $-90^{\circ}$ at the frequency of $0.273 \mathrm{THz}$, which indicates a RCP wave.
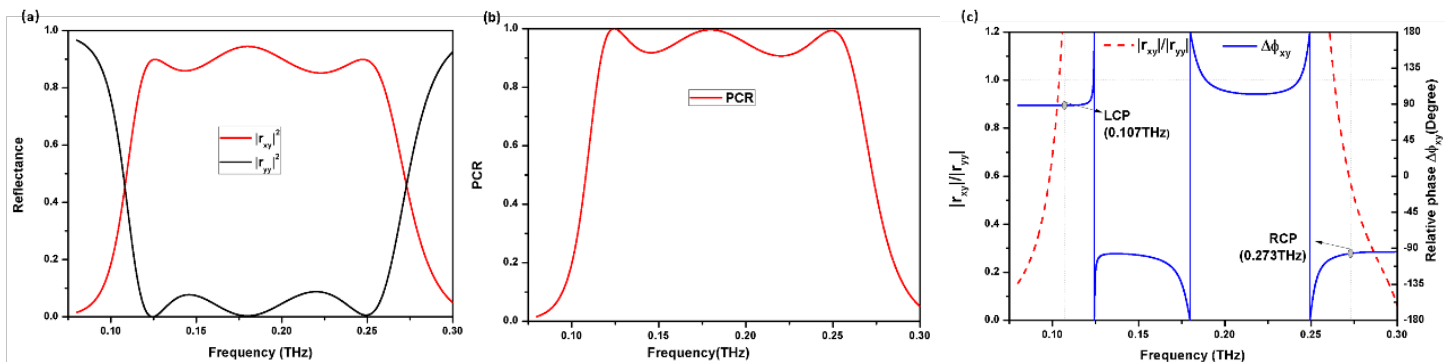

Fig. 2. Results of linear polarization conversion pattern. (a) Reflectance of $\left|r_{x y}\right|^{2}$ and $\left|r_{y y}\right|^{2}$.(b) PCR (c) Phase difference of $\Delta \phi_{x y}$ between $r_{x y}$ and $r_{y y}$

The surface current distributions for the top layer and bottom metal ground at the three resonant frequencies are demonstrated in order to better understand the physical mechanism of the polarization converter. Fig. 3(a) shows the current distributions of the top and back metal layers at the frequency of $0.123 \mathrm{THz}$. The current at the top and bottom streaming in opposite directions, and thus results in a magnetic dipole $\mathrm{m}_{1}$, which means a magnetic coupling. The y component $H_{1 y}$ of the induced magnetic field $H_{1}$ paralleled to the incident electric field $E_{y}$, which leads to a cross-polarization, the x component $H_{1 x}$ of the induced $H_{1}$ is perpendicular to the incident filed $E_{y}$, which leads to a co-polarization. The similar physical mechanism occurs at the resonant frequency of $0.18 \mathrm{THz}$, as shown in Fig. 3(b), the current on the two side arms streams in opposite directions compared with the bottom layer, and thus results in a magnetic dipole $\mathrm{m}_{2}$. At the frequency of $0.248 \mathrm{THz}$, the current on the top layer and the bottom layer result in three electric dipoles $P_{1}, P_{2}$ and $P_{3}$, as shown in Fig.3(c), which means the electric coupling, the x components $P_{1 x}, P_{2 x}$ and $P_{3}$ are perpendicular to the incident filed $E_{y}$, thus lead to cross-polarization.

Taking into account the actual situation that the EM waves not always incident onto a converter perpendicularly, hence the incident angel was changed to get different reflection results with an oblique incidence. Fig. 4 shows the different reflectance with the incident angle $\theta$ vary from $0^{\circ}$ to $45^{\circ}$. One can see that some sub-bands appear with the rising of $\theta$, this is mainly because the incident polarized direction is changed when changing the incident angle, the incident wave is no longer pure y-polarized, the polarization conversion is changed consequently. Nevertheless, the converter still keeps a broadband property when the incident angle up to $45^{\circ}$, maintaining a relatively high polarization conversion efficiency for oblique incidence.

In order to investigate the effect of dielectric thickness on the polarization conversion, the thickness t was changed from $160 \mu \mathrm{m}$ to $200 \mu \mathrm{m}$. Fig. 5 shows different reflectance and relative phase for different thickness, respectively. With the increasing of the thickness, the resonant frequencies shift toward low frequencies, meanwhile, the conversion efficiency could be changed, as shown in Fig.5 (a). Obviously, the relative phases can also be altered due to the different transmission distance, as shown in Fig.5(b). As a consequence, the proposed structure is sensitive to the change of the thickness $t$. 

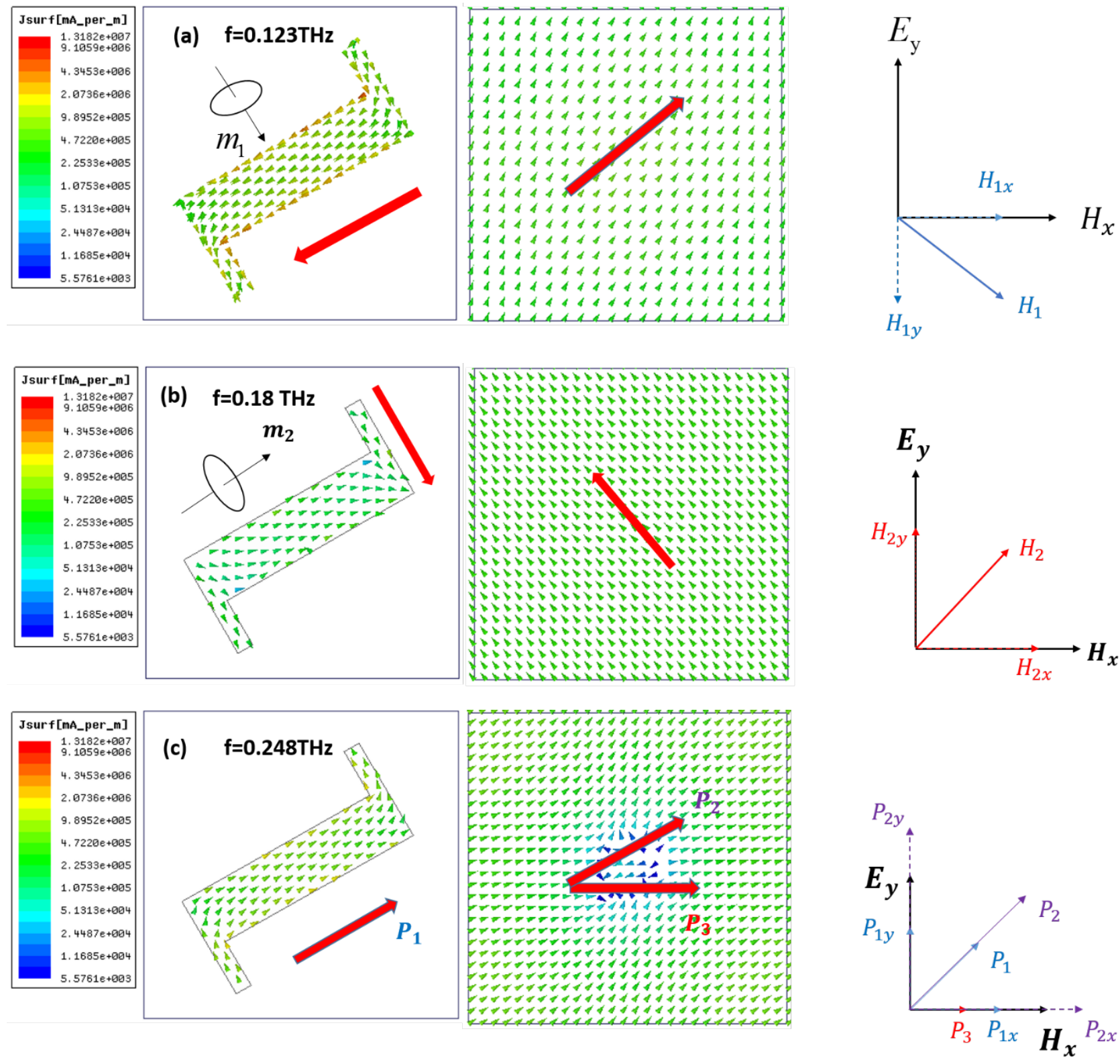

Fig. 3. Surface current distributions of the proposed polarizer. (a) at the resonant frequency of $0.123 \mathrm{THz}$. (b) at the resonant frequency of $0.18 \mathrm{THz}$. (c) at the resonant frequency of $0.248 \mathrm{THz}$.
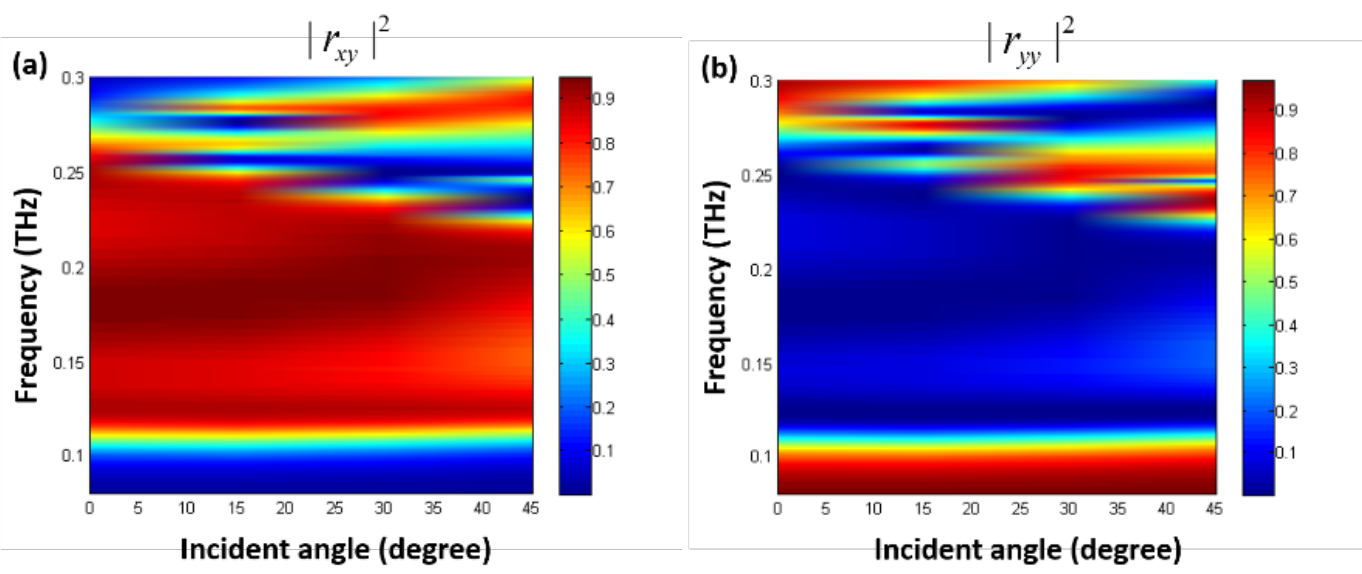

Fig. 4. Simulated results of (a) $\left|r_{x y}\right|^{2}$ (b) $\left|r_{y y}\right|^{2}$ under different incident angle from $0^{\circ}$ to $45^{\circ}$ 


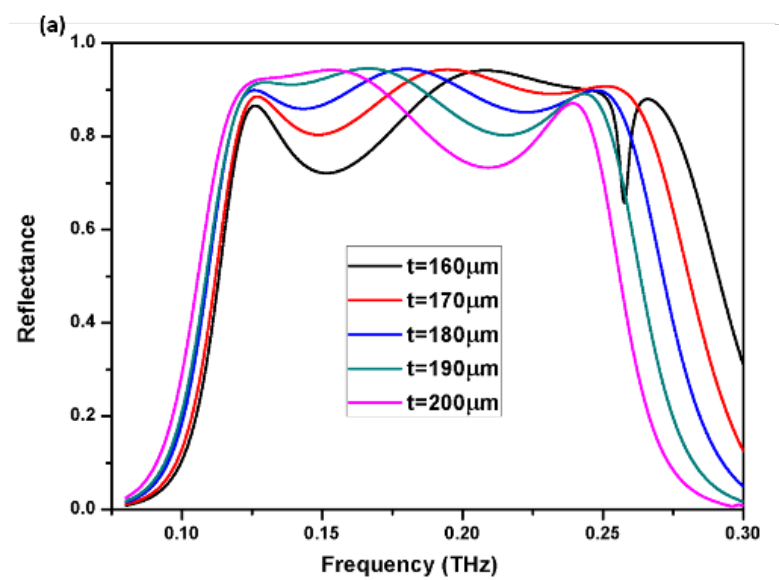

Fig. 5. Simulated results of (a) $\left|r_{x y}\right|^{2}$

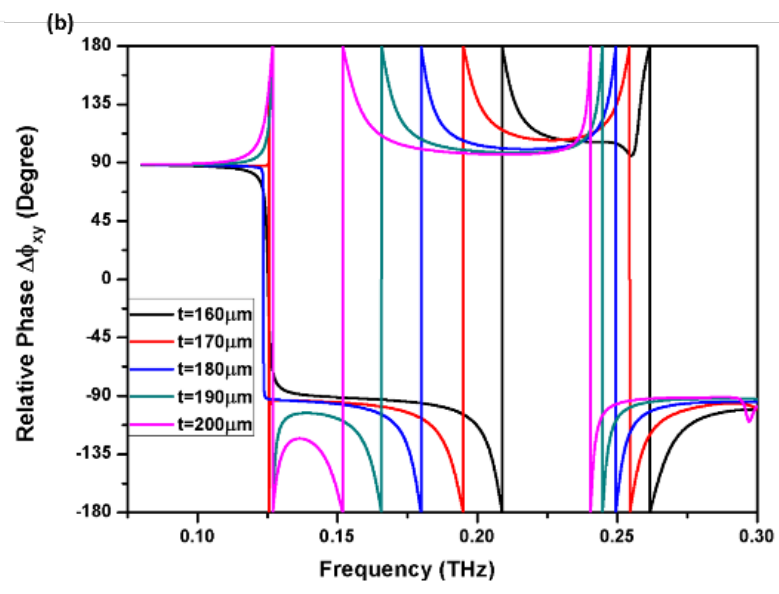

(b) $\Delta \phi_{x y}$ with different thickness

\section{Conclusion}

In summary, we propose a broadband, highly efficient and wide-angle reflective polarizer based on Z-shaped MMs at sub- $\mathrm{THz}$ frequencies. The converter can convert a LP wave to its cross-polarized wave at three resonant frequencies, which can also convert a LP wave to a CP wave at other two frequencies. The polarization conversion efficiency for normal incidence larger than 80\% is obtained from $0.116 \mathrm{THz}$ to $0.26 \mathrm{THz}$. The converter tolerances to wide angles of incidence, and shows sensitive to the change of the thickness t. With simple geometry and high performance, the proposed polarizer could be valuable in $\mathrm{THz}$ regime.

\section{Acknowledgments}

This work was supported by the National Natural Science Foundation of China (No. 91438118), and the Fundamental Research Funds for the Central Universities of China (No. ZYGX2014J037 and ZYGX2014J033).

\section{References}

[1] N. Yu, P. Genevet, M. A. Kats, F. Aieta, J. P. Tetienne, F. Capasso, et al., "Light propagation with phase discontinuities: generalized laws of reflection and refraction," Science, vol. 334, pp. 333-7, Oct 212011.

[2] Y. Jia, Y. Liu, Y. J. Guo, K. Li, and S.-X. Gong, "Broadband Polarization Rotation Reflective Surfaces and Their Applications to RCS Reduction," IEEE Transactions on Antennas and Propagation, vol. 64, pp. 179-188, 2016.

[3] D. Wang, L. Zhang, Y. Gong, L. Jian, T. Venkatesan, C.-W. Qiu, et al., "Multiband Switchable Terahertz Quarter-Wave Plates via Phase-Change Metasurfaces," IEEE Photonics Journal, vol. 8, pp. 1-8, 2016.

[4] J. Xu, T. Li, F. F. Lu, S. M. Wang, and S. N. Zhu, "Manipulating optical polarization by stereo plasmonic structure," Opt Express, vol. 19, pp. 748-56, Jan 172011.

[5] J. H. Shi, H. F. Ma, W. X. Jiang, and T. J. Cui, "Multiband stereometamaterial-based polarization spectral filter," Physical Review B, vol. 86, 2012.

[6] D. Schurig, J. J. Mock, B. J. Justice, S. A. Cummer, J. B. Pendry, A. F. Starr, et al., "Metamaterial Electromagnetic Cloak at Microwave Frequencies," Science, vol. 314, pp. 977-980, 2006.

[7] D. Lin, P. Fan, E. Hasman, and M. L. Brongersma, "Dielectric gradient metasurface optical 
elements," Science, vol. 345, pp. 2014.

[8] X. Huang, B. Xiao, L. Guo, S. Yu, and H. Yang, "Triple-band linear and circular reflective polarizer based on E-shaped metamaterial," Journal of Optics, vol. 16, p. 125101, 2014.

[9] J. Ding, B. Arigong, H. Ren, M. Zhou, J. Shao, Y. Lin, et al., "Efficient multiband and broadband cross polarization converters based on slotted L-shaped nanoantennas," Opt Express, vol. 22, pp. 29143-51, Nov 172014.

[10]H. Li, B. Xiao, X. Huang, and H. Yang, "Multiple-band reflective polarization converter based on deformed F-shaped metamaterial," Physica Scripta, vol. 90, p. 035806, 2015.

[11]L. Zhang, P. Zhou, H. Chen, H. Lu, J. Xie, and L. Deng, "Adjustable wideband reflective converter based on cut-wire metasurface," Journal of Optics, vol. 17, p. 105105, 2015.

[12]N. K. Grady, J. E. Heyes, D. R. Chowdhury, Y. Zeng, M. T. Reiten, A. K. Azad, et al., "Terahertz Metamaterials for Linear Polarization Conversion and Anomalous Refraction," Science, vol. 340, pp. 1304-1307, 2013.

[13]Y. Z. Cheng, W. Withayachumnankul, A. Upadhyay, D. Headland, Y. Nie, R. Z. Gong, et al., "Ultrabroadband reflective polarization convertor for terahertz waves," Applied Physics Letters, vol. 105, p. 181111, 2014.

[14]Z. Zhang, J. Luo, M. Song, and H. Yu, "Large-area, broadband and high-efficiency near-infrared linear polarization manipulating metasurface fabricated by orthogonal interference lithography," Applied Physics Letters, vol. 107, p. 241904, 2015. 